\title{
Proprioception and the production of adaptation and intermanual transfer to prismatic displacement
}

\author{
BENJAMIN WALLACE and LESLIE E. FISHER \\ Cleveland State University, Cleveland, Ohio 44115
}

\begin{abstract}
Proprioceptive adaptation to prismatic displacement and resultant intermanual transfer were investigated in two experiments. In Experiment 1, magnitude of adaptation and transfer were assessed as a result of the reduction of felt sensation via hypnotic anesthesia in an adapting limb. Such anesthesia reduced the magnitude of adaptation in that limb and resultant transfer in the nonadapting limb to a nonsignificant level. Such was not the case when the adapting limb was nonanesthetic. In Experiment 2, adaptation and transfer magnitude were assessed as the result of anesthetic induction in a nonadapting limb. When this was the case, adaptation was produced in the adapting limb but not in the anesthetized, nonadapting limb. The results of the two experiments generally point to proprioception as being the major source of input to the production of intermanual transfer in a prismatic adaptation task.
\end{abstract}

When subjects adapt to a prismatically displaced environment, a portion of the adaptation magnitude is represented by a component responsible for felt limb location or orientation (Harris, 1963, 1965; Hay \& Pick, 1966; Redding, 1978; Redding \& Wallace, 1976, 1978; Wallace, 1977; Wallace \& Redding, 1979; Wilkinson, 1971). This adaptation component is generally assessed in a head-hand coordination task where subjects are required to localize the straightahead position in space with an unseen limb before and after an exposure to prismatic displacement. The preexposure-postexposure localization difference value is termed a proprioceptive shift (PS) and postexposure pointing is typically in the direction opposite the exposure, prism-base orientation (Harris, 1965; Welch, 1974, 1978).

Recently, it has been shown that PS is amenable to intermanual transfer (Choe \& Welch, 1974; McLaughlin \& Bower, 1965; Wallace, 1978; Wallace \& Redding 1979; Melamed, Beckett, \& Hill, Note 1). Specifically, PS can be measured for a nonadapting limb as well as for an adapting limb. As such, the recalibrated proprioceptive input appears to influence control of the unexposed limb.

The experimental paradigm which appears to maximize intermanual transfer involves a situation where a subject is required to observe a limb perform a terminal, ballistic movement while being permitted some head movement to observe the pointing action (Wallace, 1978; Wallace \& Redding, 1979). Terminal,

The authors acknowledge the assistance of Ingelore Reschke in data collection. Reprint requests should be sent to Benjamin Wallace, Department of Psychology, Cleveland State University, Cleveland, Ohio 44115. ballistic movement permits the subject to observe a pointing action only at the conclusion of a sagittal arm reach away from the body.

If intermanual transfer does indeed involve the transference of proprioceptive input or information to the unexposed limb, it may be possible to verify this phenomenon with the aid of hypnotic anesthesia. This manipulation has previously been employed to test the role of felt sensation reduction in a prism adaptation task (Garrett \& Wallace, 1975; Wallace \& Garrett, 1973, 1975). Specifically, it has been shown that the induction of hypnotic anesthesia in an adapting arm during prism adaptation reduces the PS component to a nonsignificant level. As such, felt sensations in an adapting limb appear to be responsible for the production of the PS.

Wallace and Garrett (1973) also examined the magnitude of intermanual transfer to a nonadapting limb resulting from a paradigm where a hypnotically anesthetized, adapting limb was observed during prism exposure. Regardless of whether an adapting limb was or was not anesthetized, intermanual transfer was not produced. However, this study did not employ the conditions which have subsequently been shown to maximize the production of transfer (Wallace, 1978). As such, it was not a strong test of transfer production or reduction.

If the conditions which appear to maximize intermanual transfer are employed, it should be possible to determine if transfer is, indeed, the result of proprioceptive input. Theoretically, had Wallace and Garrett (1973) employed terminal exposure and unconstrained head movement during the observation of arm movement, one would have expected transfer when an adapting limb was capable of feeling arm- 
movement sensations. Conversely, the absence of such felt sensations should not only have eliminated PS (as it indeed did), but it also should have eliminated transferable PS. This possibility was considered in Experiment 1.

\section{EXPERIMENT 1}

\author{
Method \\ Subjects. Thirty volunteers from the introductory psychology \\ classes at Cleveland State University served as subjects. Prior to \\ experimental participation, all subjects had participated in a mass- \\ testing session where the Harvard Group Scale of Hypnotic Sus- \\ ceptibility, Form A (Shor \& Orne, 1962) was administered. Twenty \\ subjects scored high (9-12) on the test, while 10 subjects scored \\ low (0-3).
}

Apparatus. The apparatus was similar to that employed by Wallace (1978). This structure consisted of a three-layer, rectangular, wooden, box-like frame $(77 \mathrm{~cm}$ high, $62 \mathrm{~cm}$ wide, and $62 \mathrm{~cm}$ deep) open on the side facing the subject. When subjects placed one arm within the structure, on the lowest layer, the arm was not visible. In this area, during test, arm position could be noted by an experimenter by determining its location along a $180^{\circ}$ arc, calibrated in $1^{\circ}$ increments. During exposure, placement of the subject's arm upon the center layer permitted the individual to perceive arm movement only at the terminus of a response action. Observation of arm movement throughout most of the exposure session was prevented by a third layer, located $12 \mathrm{~cm}$ directly above the second layer of the apparatus. During exposure, all visible surfaces were homogeneous in appearance, e.g., there were no visible targets toward which subjects could localize arm movements. Attached to the apparatus was a Marietta Instruments combination head-and chinrest. The chin portion of the head constraint could be loosened to permit head swivel.

Procedure. Each subject was tested individually by being led to an experimental room and seated in a chair before the apparatus. The subject's head was then positioned in the head-and chinrest. One of the two experimenters (Experiment 1) then placed Risley prisms, set to 0 diopters and mounted in welder's goggles, over the subject's eyes.

The PS test required the subjects to place their dominant or nondominant hands (random starting order) on the lowest layer of the apparatus, and to point to the position in space which they believed to be straight ahead of their noses. This task was accomplished without sight of arm movement and with constrained head movement. During this preexposure, baseline determination, the straight-ahead localization task was performed five times with each hand, randomly alternating hands across the total 10 pointing trials.

Following the establishment of the PS baseline, the subjects were moved to a different room. At this point, if the subjects were judged to be high in hypnotic susceptibility by virtue of their hypnosis test scores, they either had their dominant arms hypnotically anesthetized or were requested to role-play the sensation of having their dominant limbs so anesthetized. Ten subjects participated in each of the aforementioned conditions. The subjects were sufficiently informed with respect to the consequences of induced, hypnotic anesthesia. They were told that their arms would feel totally numb and absent of all felt sensations. The highsusceptibility, role-playing group served as a control to be certain that hypnotic susceptibility, per se, was not an influencing factor in the experiment. If subjects scored low on the attribute of hypnotic susceptibility, they were requested to role-play the sensation of having their limbs hypnotically anesthetized. Ten subjects were assigned to this condition, which is traditionally employed when hypnosis is used as a manipulation. Arm dominance was determined by asking the subjects to respond to standard arm dom- inance questions (i.e., With which hand do you write? With which hand do you hold an eating utensil?, etc.). Success of the hypnotic anesthesia manipulation was determined by requesting subjects to point to their noses with their eyes closed. The ability to perform this simple, proprioceptive task has been shown to be affected by the implementation of hypnotic anesthesia in the pointing limb. When this manipulation is in effect, subjects either miss the nose location or show a prolonged reaction time from movement initiation to nose localization (Wallace \& Hoyenga, in press). This manipulation check was conducted by the second experimenter (Experimenter 2) on all subjects regardless of condition assignment. Only hypnotically anesthetized subjects responded in error to the nose-touch task. Experimenter 1 was blind with respect to subject group assignment and with respect to the anesthesia manipulation check.

Following anesthesia induction (real or role-played), the subject was returned to the original experimental room which contained the previously described apparatus. Upon return, the subject was again seated in the chair before the apparatus. At this point, Experimenter 1 placed the prisms (set to 20 diopters, base right) over the subject's eyes. The subject was then requested to point straight ahead of the nose. Pointing was performed in a ballistic fashion such that the subject extended the dominant arm away from the body and then brought it back. This back-and-forth action was performed approximately 40 times within a 2 -min exposure session. During the exposure, head movement was unconstrained and terminal pointing was performed where the consequences of a point were perceived only at the terminus of the action.

Following orism exposure, the prisms were removed from the subject's eyes and this individual was returned to the experimental room where anesthesia (real or role-played) had been induced. Experimenter 2 then removed the anesthesia from those who actually received this manipulation or informed role-playing subjects that they no longer needed to pretend that their dominant arms were hypnotically anesthetized. Following the removal of the anesthesia, which required approximately $30 \mathrm{sec}$ for completion, Experimenter 2 asked the subjects to perform again the nose-touch task. At this portion in the experiment, all subjects performed the task veridically.

Immediately after anesthesia removal, the subject was returned to the experimental room containing the apparatus, where Experimenter 1 asked each subject to perform the preexposure localization task in a postexposure session. As in the preexposure portion of the experiment, the prisms were set to 0 diopter, head movement was constrained, and arm localization actions were performed 10 times, 5 with each limb in a randomly alternating fashion. Level of adaptation was specified as the signed difference between mean preexposure and mean postexposure localization measures.

\section{Results and Discussion}

A preliminary analysis of the data indicated the absence of a significant trials effect for the straightahead localization test. Consequently, subsequent analyses were restricted to the averaged difference score between preexposure and postexposure pointing accuracy to the straight-ahead position (PS). An adaptive shift for the pointing response required postexposure pointing to be in the direction opposite prism displacement (Harris, 1965; Welch, 1978). All subjects in the present study demonstrated such an adaptive shift.

Dependent measure $t$ tests were employed to determine if the mean preexposure-postexposure difference scores (shifts) differed significantly from zero. In a 
comparison of the PS values for the adapting limb for each of the three groups of subjects, the group which received actual, hypnotic anesthesia demonstrated a preexposure-postexposure localization difference or PS of $.50^{\circ}(\mathrm{SD}=1.21)$. This value was not found to be significant $[\mathrm{t}(9)=1.37]$. However, each of the role-playing groups demonstrated significant PS values. The mean PS for the high hypnotic susceptibility, role-playing group was $2.80^{\circ}$ (SD = 1.63) $[\mathrm{t}(9)=6.83, \mathrm{p}<.001]$, while the mean PS for the low hypnotic susceptibility, role-playing group was $2.95^{\circ}(\mathrm{SD}=1.39)[\mathrm{t}(9)=7.91, \mathrm{p}<.001]$. The difference between the mean values for the roleplaying groups was not significant. However, the difference between each of the role-playing groups and the actual, hypnotic anesthesia group was significant $(\mathrm{p}<.005)$.

In terms of PS transfer to the nonadapting limb, the mean PS values for the hypnotic anesthesia group, the high hypnotic susceptibility, role-playing group, and the low hypnotic susceptibility, role-playing group were $.12^{\circ}(\mathrm{SD}=.56), 1.19^{\circ}(\mathrm{SD}=1.05)$, and $1.22^{\circ}(\mathrm{SD}=.84)$, respectively. When unexposed arm adaptation (PS transfer) was compared to exposed arm adaptation and converted to a percentage value, the induction of anesthesia in an adapting limb did not result in significant production of PS transfer. However, the percent of transfer for each of the other conditions was significant: $42.50 \%$ for high hypnotic susceptibility, role-playing subjects $[\mathrm{t}(9)=4.87$, $\mathrm{p}<.001]$ and $41.36 \%$ for low hypnotic susceptibility, role-playing subjects $[\mathrm{t}(9)=4.67, \mathrm{p}<.001]$. Significant differences were found between percent of transfer in each of the role-playing conditions and the actual hypnotic anesthesia condition $(p<.001)$.

With respect to the effect of anesthesia induction in an adapting limb on subsequent adaptation production, the results of this experiment corroborate the findings of previous studies (Garrett \& Wallace, 1975; Wallace \& Garrett, 1973, 1975). Specifically, when felt sensations are reduced in an adapting limb, adaptation to prismatic displacement is reduced to a nonsignificant level. Thus, felt sensation appears to be a requisite for the production of a proprioceptive shift (PS). Such results support Harris' (1965) theory that adaptation to a displaced visual field involves a change in the felt position of the arm relative to the body.

In terms of intermanual transfer, it is not logical to expect such when adaptation was not produced in the opposite limb. Since no transfer was produced in Experiment 1 when an adapting limb was anesthetized, it is possible to theorize at this point that proprioception or proprioceptive information concerning arm location with respect to the straightahead position in space and with respect to body position did not transfer to the nonadapting limb when this limb was not capable of receiving such input.

However, before it can be concluded that felt sensation and proprioception are the major sources of information in the production of intermanual transfer, a second experiment is necessary. Thus, if felt sensation reduction or elimination in an adapting limb also reduces or eliminates intermanual transfer as measured in a nonadapting limb, such transference might also be reduced if a nonadapting limb were prevented from experiencing normal, felt sensations during an adaptation period. If a prismatically viewed, adapting limb does experience felt sensations during arm movement, PS should be produced. However, if such sensations are reduced in the unexposed, nonadapting limb while the exposed limb is adapting, transfer should be considerably reduced. This should be the case if proprioceptive input normally would transfer from the adapting limb to the unexposed, nonadapting limb but is being blocked or prevented by virtue of the unexposed limb not being capable of receiving veridical, felt sensations (proprioception) concerning its location with respect to the body. Experiment 2 was concerned with testing this possibility.

\section{EXPERIMENT 2}

\section{Method}

Subjects. Twenty volunteers from introductory psychology sections served as subjects. Prior to experimental participation, as in Experiment 1, all individuals participated in a mass-testing session where 10 of the subjects scored high (9-12) and 10 scored low (0-3) on the Harvard Group Scale of Hypnotic Susceptibility, Form A.

Apparatus. The apparatus was the same as that employed in Experiment 1.

Procedure. As in Experiment 1, each subject was tested individually. The procedure was similar to that employed in Experiment 1, with two major exceptions. In Experiment 1, three groups of subjects were employed: a group who received hypnotic anesthesia in an adapting limb, a group who role-played the reception of such anesthesia and who had scored high in hypnotic susceptibility, and a group who role-played the reception of hypnotic anesthesia and who scored low in hypnotic susceptibility. Since a significant difference was not found in adaptation or intermanual transfer magnitude as a function of hypnotic susceptibility level, only one group of role-playing subjects was employed in Experiment 2. All of these individuals had scored low in hypnotic susceptibility. The second group of subjects in Experiment 2 consisted of individuals who had scored high in hypnotic susceptibility and, as a result, were hypnotically anesthetized in a limb during prism adaptation.

The second difference between the procedures of Experiment 1 and Experiment 2 was the locus of hypnotic anesthesia. In Experiment 1, such anesthesia was induced in the subject's adapting limb prior to participation in a 2 -min terminal exposure to prismatic displacement. In Experiment 2, the adapting limb was not affected in terms of felt sensations. Rather, the nonadapting limb was hypnotically anesthetized. As in Experiment 1, the nose-touch task was employed to determine the success of the anesthesia induction. All subjects who were anesthetized demonstrated proprioceptive errors similar to those described for the nose-touch 
task in Experiment 1. Also, as in Experiment 1, two experimenters were employed: one who conducted all preexposure, exposure, and postexposure manipulations and assessments, and one who induced hypnotic anesthesia prior to the exposure session and who removed such thereafter. The first experimenter was blind with respect to subject group assignment.

\section{Results and Discussion}

As in Experiment 1, a preliminary analysis of the data indicated the absence of a significant trials effect for the localization task. Therefore, subsequent analyses were concerned only with averaged difference scores between preexposure and postexposure arm localizations with respect to the straight-ahead position (PS). Also, as in Experiment 1, all subjects demonstrated an adaptive shift in the correct direction, e.g., opposite the direction of prismatic displacement. Dependent $t$ tests were employed to determine if such shifts differed significantly from zero.

With respect to adapting limb PS production, each group of subjects demonstrated a significant shift. The mean PS values were $3.89^{\circ}(\mathrm{SD}=2.05)$ [ $\mathrm{t}(9)$ $=7.87, \mathrm{p}<.001]$ for the group who received anesthesia in the nonadapting limb, and $4.29^{\circ}$ (SD = 2.18) $[\mathrm{t}(9)=6.05, \mathrm{p}<.001]$ for the role-playing group. These values did not differ significantly from each other. Thus, when hypnotic anesthesia was not induced in the adapting limb, adaptation magnitude, as measured by PS, was equivalent for both groups of subjects.

However, when anesthesia was induced in the nonadapting limb, intermanual transfer was affected. The magnitude of produced intermanual transfer for the anesthesia and role-playing groups was $.59^{\circ}$ (SD $=.36)$ and $2.53^{\circ}(\mathrm{SD}=1.61)$, respectively. Only the latter value when transformed was equivalent to $58.97 \%$ transfer.

These results support the conclusion reached in Experiment 1 , namely that proprioception or felt sensation appears to play a major role in the production of intermanual transfer. When an unexposed and nonadapting limb is anesthetized during a period when the other limb is adapting proprioceptively to a prismatically displaced environment, the nonadapting limb does not receive information input concerning arm location of the adapting limb with respect to the straight-ahead position. The result is a marked reduction in intermanual transfer of PS compared to such production in Experiment 1. Therefore, the production of intermanual transfer requires that both the adapting limb and the nonadapting limb be capable of experiencing normal, felt sensations during an adaptation period. When either arm experiences a reduction in felt sensation as the result of induced hypnotic anesthesia, intermanual transfer is also reduced.

In addition to the felt sensation explanation of intermanual transfer set forth here, it has also been suggested (Hamilton, 1964) that such transfer may be mediated by a change in felt head position. If one assumes that this may be possible, then a link between induced limb anesthesia and the conditions productive of a change in felt head position must exist. Unconstrained head movement might be such a link, since this factor appears to be critical for the occurrence of intermanual transfer. Therefore, it is important to establish whether subjects in the anesthetic conditions produced head movements of lower amplitude, or were less likely to move the head than were subjects in the other condition. In other words, did the condition of induced limb anesthesia serve to constrain head movements, thereby limiting the possibility of finding transfer? At least in the present investigation, evidence for such head-movement constraint was not observed. Thus, the felt head position influence on the production of intermanual transfer as it was assessed here would appear to be minimal, at best.

\section{REFERENCE NOTE}

1. Melamed, L. E., Becket, P. A., \& Hill, G. Individual differences in components of prism adaptation derived from the ter. minal exposure, distributed practice paradigm. Paper read at the meetings of the Midwestern Psychological Association, Chicago, May 1976.

\section{REFERENCES}

Choe, C. S., \& Welch, R. B. Variables affecting the intermanual transfer and decay of prism adaptation. Journal of Experimental Psychology, 1974, 102, 1076-1084.

GarretT, J. B., \& Wallace, B. A novel test of hypnotic anesthesia. International Journal of Clinical and Experimental Hypnosis, 1975, 23, 139-147.

Hamilton, C. R. Intermanual transfer of adaptation to prisms. American Journal of Psychology, 1964, 77, 457-462.

Hargis, C. S. Adaptation to displaced vision: Visual, motor, or proprioceptive change? Science, 1963, 140, 812-813.

Harris, C. S. Perceptual adaptation to inverted, reversed and displaced vision. Psychological Review, 1965, 72, $419-444$.

HaY, J. C., \& Pick, H. L., JR. Visual and proprioceptive adaptation to optical displacement of the visual stimulus. Journal of Experimental Psychology, 1966, 71, 150-158.

McLaughlin, S. C., \& Bower, J. L. Selective intermanual transfer of adaptive effects during adaptation to prisms. Psychonomic Science, 1965, 3, 69-70.

Redding, G. M. Additivity in adaptation to optical tilt. Journal of Experimental Psychology: Human Perception and Performance, $1978,4,178-190$.

Redding, G. M., \& Wallace, B. Components of displacement adaptation in acquisition and decay as a function of hand and hall exposure. Perception \& Psychophysics, 1976, 20, 453-459.

Redding, G. M., \& Wallace, B. Sources of overadditivity in prism adaptation. Perception \& Psychophysics, 1978, 24, 58-62.

ShoR, R. E., \& ORNE, E. C. Harvard Group Scale of Hypnotic Susceptibility: Form A. Palo Alto, Calif: Consulting Psychologists Press, 1962.

WALLACE, B. Stability of Wilkinson's linear model of prism adaptation over time for various targets. Perception, 1977, 6, 145-151. 
WALlace, B. Visuomotor coordination and intermanual transfer for a proprioceptive reaching task. Journal of Motor Behavior, $1978,10,139-147$.

Wallace, B., \& Garkett, J. B. Reduced felt arm sensation effects on visual adaptation. Perception \& Psychophysics, 1973, 14, 597-600.

Whllace, B., G Garrett, J. B. Petceptual adaptation with selective reductions of felt sensation. Perception, [975, 4, 437-445.

Wallace, B, \& Hoyenga, K. B. Production of proprioceptive errors with induced hypnotic anesthesia. International Journal of Clinical and Experimental Hypnosis, in press.
Wallace, B., \& Redding, G. M. Additivity in prism adaptation as manifested in intermanual and interocular transfer. Perception \& Psychophysics, 1979, 23, 133-136.

WELCH, R. B. Research on adaptation to rearranged vision: 1966-1974. Perception, 1974, 3, 367-392.

Welch, R. B. Perceptual modification: Adapting to altered sensory environments. New York: Academic Press, 1978.

Wizkinson, D. A. Visuai-motor controi bop: A inear system? Journal of Experimencal Psychology, 1971, 89, 250-257.

(Received for publication January 30, 1979; revision accepted May 23, 1979.) 